Perspectiva Geográfica

ISSN 0123-3769 (Impreso) - 2500-8684 (En línea)

Vol. 21 No. 1 de 2016

Enero - Junio

pp. 33-62

\title{
Modelo climático de los páramos de la cordillera Oriental colombiana aplicado a regímenes de temperatura del suelo ${ }^{1}$
}

\author{
Climate Model of the Moorlands of the Colombian Eastern \\ Mountain Range Applied to Soil Temperature Regimes
}

Carlos Enrique Castro Méndez ${ }^{2}$

Yolima del Carmen Agualimpia Dualiby ${ }^{3}$

Fernando Sánchez Sánchez ${ }^{4}$

Para citar este artículo utilice el nombre completo así:

Castro, C., Agualimpia, Y. \& Sánchez, F. (2016). Modelo climático de los páramos de la cordillera Oriental colombiana aplicado a regímenes de temperatura del suelo. Perspectiva Geográfica, 21(1), 33-62.

\section{Resumen}

La aplicación (con criterios consistentes) de los regímenes de temperatura del suelo no había sido necesaria y decisiva, hasta ahora, ya que se consideraba suficiente esta información de carácter descriptivo. La temperatura asignada

1 Este artículo hace parte del proyecto de investigación 'Propuesta metodológica para la zonificación climática a diferentes escalas en Colombia, con fines de manejo sostenible del territorio. Fase l'. Grupo de Investigación PROGASP, Universidad Distrital Francisco José de Caldas, Bogotá. Colombia. Centro de Investigaciones y Desarrollo Científico -CIDC- de la Universidad Distrital Francisco José de Caldas.

2 Magíster en Geografía, Convenio UPTC-IGAC. Profesional Subdirección de Agrología - Instituto Geográfico Agustín Codazzi. cecastro77@gmail.com

3 Doctora en Ciencias Técnicas. Instituto Superior Politécnico José Antonio Echeverría. Docente Universidad Distrital Francisco José de Caldas. yagualimpiadualiby@gmail.com

4 Doctor en Ciencias Técnicas. Instituto Superior Politécnico José Antonio Echeverría. Docente Universidad Distrital Francisco José de Caldas. fersasanchezphd01@gmail.com 
al suelo provenía de la reclasificación directa del clima ambiental al edáfico con valores medios anuales dados a cada franja altitudinal hace más de 200 años; esto no concuerda con los cambios que desde la geografía biofísica se han percibido en el ambiente. Se propone un modelo climático basado en isotermas del suelo, para determinar los regímenes de temperatura a partir de información bajo coberturas vegetales y del suelo, para cada vertiente montañosa en particular. Se encontraron gradientes de temperatura $\left({ }^{\circ} \mathrm{C} / 100\right.$ m) de 0,73 y 0,61 en la vertiente occidental y oriental, respectivamente. La temperatura del suelo fue $1.9^{\circ} \mathrm{C}$ mayor que la del aire y se delimitaron los regímenes térmicos del suelo cryico, isofrígido e isomésico.

Palabras clave: isotermas del suelo, modelo climático, páramos, régimen de temperatura.

\begin{abstract}
The application (with consistent criteria) of soil temperature regimes has not been necessary and decisive so far, because this descriptive information was considered sufficient. The temperature assigned to the ground came from direct reclassification of the edaphic environmental climate with average annual values given to each altitudinal strip more than 200 years ago, which today is not consistent with the changes, have been perceived through biophysical geography. A climate model, based on isotherms, is proposed to determine soil temperature regimes from information under vegetation cover and soil for each particular mountain slope. Temperature gradients $\left({ }^{\circ} \mathrm{C} / 100 \mathrm{~m}\right)$ of 0.73 and 0.61 were found in the western and eastern slopes, respectively, of the Colombian Eastern Mountain Range Moorlands. Soil temperature was $1.9^{\circ} \mathrm{C}$ higher than air temperature, and soil temperature regimes were defined as cryic, isofrigid and isomesic.
\end{abstract}

Keywords: isotherms soil, temperature regime, climate model. 


\section{Introducción}

En Colombia, los regímenes de temperatura se determinan de acuerdo con la reclasificación de cotas altitudinales, debido a que en los trabajos de campo no se obtienen datos de temperatura del suelo, la cual juega un papel importante en la delimitación de los ecosistemas de páramo; de su correcta aplicación depende la protección y preservación de estos paisajes localizados en la zona montañosa.

Ante la diversidad de climas que tiene Colombia en la zona de montaña, el Instituto Geográfico Agustín Codazzi generó la subdivisión en la franja de clima frío propuesta por Francisco José de Caldas; las cuatro unidades fueron denominadas frío, muy frío, extremadamente frío y nival; su utilidad ha sido demostrada en estudios del suelo desde los años setenta. En las zonas bajas de montaña se mantiene la categoría dada por Caldas: pisos cálido y templado, o medio, como se denominaba hasta los años noventa.

Los pisos térmicos se relacionan espacialmente mediante la delimitación de cotas de altitud que, a su vez, representan un rango de tem- peraturas del aire, las cuales son aplicadas de manera indiscriminada en las vertientes colombianas. En este artículo se analizan los resultados de investigadores reconocidos en el tema, como Stanescu y Díaz (1971), Flórez (1986), Pabón, Eslava y Gómez (2001), que sirven de soporte para generar un modelo climático que se ajuste a los requerimientos de una cartografía semidetallada y, de esta manera, contribuir a la delimitación de las zonas que deben protegerse.

En los estudios de suelos realizados por el Instituto Geográfico Agustín Codazzi (IGAC) en sectores montañosos, los pisos térmicos suponen una correspondencia directa con los regímenes climáticos del suelo; es así como las franjas frías se consideran como régimen de temperatura del suelo isomésico; en las muy frías se denominan isofrígido, y en los pisos nivales llevan por nombre cryico. La delimitación de los pisos térmicos, según las cotas, no es aplicable a la escala semidetallada, ni constituye información técnica consistente, debido a que las vertientes montañosas presentan gradientes de temperatura diferentes por efecto del tiempo de exposición de las laderas al sol, o por la influencia de los vientos en 
la condición de humedad (Pabón, Eslava y Gómez, 2001).

Es necesario replantear el procedimiento para delimitar los páramos, de acuerdo con los requerimientos actuales del Instituto de Investigación en Recursos Biológicos Alexander Von Humboldt, con lo cual surgen nuevas propuestas en la definición de la temperatura del suelo a la escala 1:25.000. Dentro de este contexto, para establecer el límite de ecosistemas de páramo se acude a la identificación del régimen de temperatura del suelo denominado isofrígido, que corresponde a valores promedios anuales entre $4{ }^{\circ} \mathrm{C}$ y $8{ }^{\circ} \mathrm{C}$.

La pregunta que orienta la investigación es: ¿Cómo generar los regímenes de temperatura del suelo en páramos de la cordillera Oriental y aproximarse a la escala de representación semidetallada sin utilizar los pisos térmicos?

En este artículo se presentan los resultados obtenidos desde los siguientes objetivos específicos: analizar el procedimiento utilizado para determinar el régimen de temperatura del suelo a nivel semidetallado; revisar el procedimiento que se aplica en Colombia para definir el clima edáfico, y diseñar un procedimiento para definir el régimen isofrígido en un sector de páramo de la cordillera Oriental, con el propósito general de precisar la delimitación de los pisos térmicos en Colombia $\mathrm{y}$, especialmente, el régimen de temperatura del suelo, ya que en los procesos utilizados en la Subdirección de Agrología del Instituto Geográfico Agustín Codazzi estos límites provienen de la asignación de intervalos de temperatura con base en cotas de altitud (IGAC, 2014a).

En la revisión de documentos técnicos relacionados con el clima como factor formador del suelo no se encontraron procedimientos que definan las unidades climáticas ni especificaciones para determinar los regímenes de temperatura del suelo; la mayor parte de las experiencias existentes en este tema tienen más de diez años, razón por la cual debe retomarse a la luz de nuevos problemas que puedan generarse en el ambiente.

La hipótesis planteada en la presente investigación es la siguiente: es posible delimitar los regímenes térmicos en páramos de la cordillera Oriental, mediante un modelo climático basado en isotermas del suelo. 
Los cambios climáticos que acontecen en el presente pueden generar variaciones en los límites de las isotermas del suelo; por tanto, los modelos climáticos que relacionaban la altitud con la temperatura, cuyo referente corresponde aproximadamente a 200 años de antigüedad, no deben tomarse como base para el monitoreo del calentamiento global; se requieren ajustes a los procedimientos que eran válidos para la escala general.

\section{Planteamiento del problema}

No hay referencias de la aplicación de modelos climáticos en los levantamientos de suelos en Colombia, y en otros países se ha tratado el tema de manera tangencial, lo cual origina imprecisiones cuando se piensa reclasificar el clima del suelo a partir de lo ambiental; por tal razón, se requiere mejorar el procedimiento para ampliar su escala de representación.

En el manual de reconocimiento de suelos de Australia (McKenzie, Grundy, Webster, \& Ringrose-Voase, 2008) el clima se estudia como un factor que interactúa con las propiedades físicas del suelo; este análisis combinado ofrece características que pueden considerarse según la interacción de factores que influyen en la formación de suelos, bajo el supuesto de que los procesos que les dieron origen se conservan aún en el presente.

The National Committee on Soil and Terrain (2009) considera el clima como generador de coberturas vegetales que inciden directamente en la formación de suelos, al igual que el paisaje y el sustrato geológico. Todos los esfuerzos los dirige a realizar una descripción detallada de las coberturas vegetales, pasando por análisis escalar de formaciones vegetales, estructura, composición florística y descripción de especies dominantes. El clima se asume por las coberturas vegetales, que son su producto, y no por el análisis de series históricas de precipitación y temperatura.

Latemperaturadelsueloinfluyeenel desarrollo de raíces, disponibilidad de nutrientes y movimiento del agua, cambia según la profundidad y presenta mayor variabilidad en la superficie del suelo, con lo cual establece un círculo (de calentamiento y enfriamiento) que permite la meteorización y desarrollo genético de este. Los factores que afectan la temperatura están relacionados con la cantidad 
de energía proveniente del sol, la cantidad absorbida según su color, el tipo de cobertura, la humedad, la pendiente y el ángulo de incidencia de los rayos solares. El factor de mayor influencia en la temperatura del suelo se relaciona con la exposición de la ladera al sol; los suelos con exposición hacia el sur se calientan más en la primavera que los suelos expuestos hacia el norte (Milo, Save y Bennett, 1997).

El manual de levantamiento de suelos (USDA, 1993) menciona el clima solo como su factor formador, y lo analiza a través del efecto combinado con la vegetación; la FAO (2012) plantea la utilidad de trabajar con datos de temperatura y precipitación media mensual en el sitio del perfil del suelo y orienta el tema del clima al análisis de períodos de crecimiento vegetal mediante la cuantificación de días en los cuales la precipitación excede la evapotranspiración. En el manual USDA-NRCS (2012) se mencionan los regímenes de temperatura del suelo y se hace distinción de las temperaturas de este en las zonas intertropicales, cuyo gradiente de temperatura invierno-verano no supera los $6^{\circ} \mathrm{C}$. La clave taxonómica de suelos USDA-NRCS (2014) no presenta avances en cuanto a la de- terminación de los regímenes de temperatura del suelo.

La metodología para levantamiento de suelos (IGAC, 2014a) y el Manual de Códigos del Instituto Geográfico Agustín Codazzi (IGAC, 2014b), no tienen establecidos los procedimientos para delimitar los regímenes de temperatura del suelo; las tablas de referencia que contienen los instrumentos técnicos colombianos siguen pautas dadas por los manuales de campo del Departamento de Agricultura de los Estados Unidos (USDA, 1993), y en Colombia no se han realizado los ajustes correspondientes a la escala semidetallada; en tal caso, existe la posibilidad de definir la temperatura del suelo mediante un procedimiento general que la relaciona con el piso térmico. Aun cuando el procedimiento es adecuado para escalas generales, debe revisarse para estudios semidetallados y detallados, con el fin de obtener delineaciones de suelos con mayor nivel de detalle.

La metodología para levantamientos de suelos (IGAC, 2014a) menciona el clima como un paso que se sigue durante la recolección y evaluación de la información de la zona de estudio, no plantea la utilidad de 
este factor en la identificación de los suelos y lo menciona como un referente para el trabajo de campo. Se encuentra mayor información sobre la utilidad del clima en los levantamientos de suelos en el capítulo del medio biofísico de la Guía para elaborar la memoria técnica (IGAC, 2014c), aunque en ella también se le menciona en la parte descriptiva, se pide referenciar los elementos climáticos precipitación, temperatura, humedad relativa y brillo solar, con la alternativa de utilizar otras variables para definir los pisos térmicos y la condición de humedad; la guía plantea el análisis climático temporal mediante balances hídricos que muestren déficit o excesos.

El tema de zonificación climática de la Subdirección de Agrología del Instituto Geográfico Agustín Codazzi está resumido en el documento Zonificación Climática (IGAC, 2014d), en el cual se nombran tres clasificaciones sin especificar cuál de ellas es la que se aplica en el levantamiento de suelos: Caldas-Lang, Martonne y la adaptación de Zonas de Vida (la más utilizada), mediante la cual se definen los pisos térmicos y se combinan con los índices de hume- dad de Holdridge para determinar la unidad climática.

Los modelos climáticos utilizados para delimitar los páramos son variados y tienen como referente, principalmente, la huella del clima en los paisajes junto con la presencia de estratos arbustivos dominantes; algunos esquemas son tan simples que proponen los límites de páramo de acuerdo con la presencia de frailejones (Espeletia sp.) y pajonales (Calamagrostis efusa); la identificación de especies indicadoras del ecosistema no es aplicable a los páramos de la cordillera Oriental, debido a la alta intervención en estos paisajes y a la diversidad de frailejones y de otras especies que se encuentran asociadas a la posición en el paisaje; también se considera que una línea de páramo definida por una cota es insuficiente, pues estos ecosistemas presentan diferencias de temperatura al menos en dos de sus vertientes.

Los modelos climáticos que han sido utilizados para diferenciar los paisajes de alta montaña y que eventualmente pueden aplicarse en la delimitación de áreas de páramo se referencian a continuación: 
Según Flórez (2003), el sistema cordillerano colombiano es producto del fenómeno tectónico sucedido a escala mundial, y tuvo como efecto un cambio bioclimático en el sentido altitudinal; los periodos glaciares acumularon potentes masas de hielo modeladoras de los paisajes, y el ascenso de la cordillera posibilitó un incremento en el potencial gravitatorio, generando cambios bioclimáticos que ayudaron en la configuración del relieve actual.

El trabajo realizado por el Instituto de Hidrología, Meteorología y Estudios Ambientales (IDEAM, 2010), denominado Sistemas morfogenéticos del territorio colombiano escala 1:500.000, presenta la delimitación de siete unidades bioclimáticas con las cuales identifica y caracteriza los procesos geológicos que actuaron como modeladores del relieve; el esquema utilizado por el IDEAM propone la interacción de procesos tectónicos y coberturas vegetales en unidades basales distribuidas en el paisaje de montaña.

El modelo climático para los temas relacionados con las coberturas vegetales se basa en cotas de altitud que varían en relación con las uti- lizadas en el Instituto Geográfico Agustín Codazzi; en efecto, para los análisis geográficos y ambientales realizados en el IDEAM se nombran como pisos bioclimáticos y se incluyen las siguientes cotas altitudinales: ecuatorial, menor de $1.000 \mathrm{msnm}$; subandino, de 1.000 msnm a $2.300 \mathrm{msnm}$; andino, de $2.300 \mathrm{msnm}$ a $3.000 \mathrm{msnm}$ (variación de más o menos $100 \mathrm{~m}$, según la vertiente); altoandino, de 3.000 msnm a 3.800 msnm (con variación de más o menos $200 \mathrm{~m}$ entre vertientes); páramo, de $3.800 \mathrm{msnm}$ a $4.800 \mathrm{msnm}$ (variación de más o menos 100 m según la vertiente); superpáramo de $4.300 \mathrm{msnm}$ a $5.200 \mathrm{msnm}$ (límite inferior de las glaciares), y glaciar, mayor de 4.800 msnm, según la vertiente.

Las cotas altitudinales de la Sierra Nevada de Santa Marta fueron trabajadas como pisos morfoclimáticos por Bartels (1984) y por Flórez (2003), quienes tomaron las cotas altitudinales como variables determinantes en la modelación de los paisajes colombianos, y en ninguno de los dos trabajos se distinguieron vertientes montañosas cálidas o frías.

Muy pocas investigaciones con relación a modelos climáticos se re- 
portan en el mundo. En Colombia, Narváez y León (2001) justifican las diferencias de gradiente de temperatura por cotas altitudinales para cada una de las cordilleras colombianas y algunas regiones, tomando como referencia la circulación de los vientos alisios, el sistema de mesoescala del Pacífico y la zona de convergencia intertropical.

Se distinguen, básicamente, tres modelos climáticos aplicados a sistemas montañosos, los cuales pueden ser analizados según la escala. El primero es el biogeográfico, propuesto por Holdridge (Espinal, 1978), cuyos límites fueron trazados de acuerdo con la biotemperatura, en la cual se incluye el efecto de las coberturas vegetales dominantes y la posición geográfica medida en la latitud; por tanto, este método se considera de escala global. Una nueva interpretación del clima de montaña en Colombia, o segundo modelo, lo propuso Cuatrecasas (1958) y fue ampliado por Reyes et al. (1995), retomando la denominación de ecosistema o piso bioclimático; en él se ajustaron las cotas de altitud según la región natural colombiana y la fisiología de las plantas identificadas en campo. La propuesta de ampliar la división de pisos térmicos propuesta por Caldas, en el sector de montaña (tercer modelo climático), la realizó el Instituto Geográfico Agustín Codazzi (IGAC, 1981; 2014d), para distinguir otras unidades climáticas en el piso térmico frío.

Los tres modelos climáticos expuestos basan sus límites en cotas de altitud y presentan diferencias entre sí. La propuesta consiste en mantener los valores de temperatura designados para cada altitud o piso térmico (IGAC, 2014d) y realizar la delimitación mediante el sistema de isotermas basadas en la correlación existente entre temperatura del suelo y del aire tomada en campo (Agualimpia y Castro, 2016); lo anterior genera una redefinición de cotas para los pisos térmicos $\mathrm{y}$, especialmente, se realiza una interpretación directa de los regímenes de temperatura del suelo.

La actualización reciente de la cartografía de clima en Colombia, realizada por el IDEAM, incluye un modelo climático para montaña que difiere en sus cotas del Manual de procedimientos de la zonificación climática (IGAC, 2014d). IDEAM, IGAC, IAVH, INVEMAR, SINCHI e IIAP (2007) presentaron la delimitación de pisos 
térmicos cálido y templado con una disminución de 200 metros en su cota de altitud para toda Colombia, acorde con el nuevo enfoque ambiental y la implementación de nuevos procedimientos digitales basados en modelos de regresión; en contraste, también se había generado el Atlas climatológico (IDEAM, 2005), que mantenía las mismas cotas de altitud de los pisos térmicos que trabaja el Instituto Geográfico Agustín Codazzi. La variación de cotas en los dos informes puede entenderse como una aplicación para escalas de representación diferentes.

Informes expuestos en varios eventos internacionales, como el I Congreso de Cambio Climático, realizado en Guatemala en el 2014, indican incremento en la temperatura a nivel global, lo cual, cartográficamente, generaría un incremento en la cota de los pisos térmicos y no un descenso, como muestra el mapa de clima de Colombia más reciente. La preocupación por el descenso de las lluvias lleva a problemas de variabilidad climática en amplios sectores de la Tierra y, como consecuencia, se generan temperaturas extremas que pueden generar cambios en las cotas que presentan la temperatura índice para cada piso térmico.

\section{Marco teórico}

Caldas modificado (IGAC, 2014d) establece para la montaña colombiana cuatro franjas altitudinales de categorías: frío, muy frío, extremadamente frío y nival; a estas franjas altitudinales les corresponde un rango de temperaturas del aire (Tabla 1). En las altitudes establecidas para cada piso térmico se contempla una variación de \pm 400 metros de altitud (IDEAM, 2005); esto muestra la posibilidad de que haya una variación en la franja altitudinal a causa de las condiciones locales; una nueva interpretación sería dar la posibilidad de que el dato de temperatura y las isotermas del suelo sean las que definan la franja altitudinal que tiene cada piso térmico.

Los regímenes de temperatura del suelo son características diagnósticas del sistema taxonómico de suelos del United States Department of Agriculture (USDA)-Natural Resources Conservation Service (NRCS) (USDA-NRCS, 2014) y están relacionados con valores promedio de temperatura que en Colombia se asocian a los pisos térmicos. Debido a la subdivisión que ha realizado del piso frío el IGAC (2014d), la temperatura de 
aire no tiene completos los datos correspondientes de temperatura del suelo para hacer una reclasificación adecuada (Tabla 1).

Tabla 1. Pisos térmicos y regímenes de temperaturas para escalas generales

\begin{tabular}{|l|c|l|c|c|}
\hline \multicolumn{1}{|c|}{ Piso térmico } & \multicolumn{1}{|c|}{$\begin{array}{c}\text { Altitud } \\
(\mathbf{m})\end{array}$} & $\begin{array}{c}\text { Régimen de } \\
\text { temperatura } \\
\text { del suelo }\end{array}$ & $\begin{array}{c}\text { Temperatura } \\
\text { del aire }{ }^{\circ} \mathbf{C}\end{array}$ & $\begin{array}{c}\text { Temperatura } \\
\text { del suelo } \\
{ }^{\mathbf{0}} \mathbf{C}\end{array}$ \\
\hline Nival & 4.700 a 5.775 & Gélico & menor a 1,5 & menor a 0 \\
\hline Subnival & 4.200 a 4.700 & Cryico & 1.5 a 4 & - \\
\hline $\begin{array}{l}\text { Extremadamente } \\
\text { frío }\end{array}$ & 3.600 a 4.200 & Cryico & 4 a 8 & 0 a 8 \\
\hline Muy frío & 3.000 a 3.600 & Isofrígido* & 8 a 12 & - \\
\hline Frío & 2.000 a 3.000 & Isomésico & 12 a 18 & 8 a 15 \\
\hline Templado & 1.000 a 2.000 & Isotérmico & 18 a 24 & 15 a 22 \\
\hline Cálido & menor a 1.000 & Isohipertérmico & mayor a 24 & mayor a 22 \\
\hline
\end{tabular}

*El régimen térmico isofrígido corresponde al régimen cryico con verano más cálido.

Fuente: Elaboración propia con base en IGAC- ICA (1985, 2010), USDA-NCRS (2014).

La localización de Colombia en la zona ecuatorial, con pocas variaciones de calor por incidencia vertical del sol durante casi todo el año, permite indagar sobre la variación de la temperatura del suelo durante el día, dado que en la actualidad el régimen isofrígido se asigna a zonas localizadas a altitudes entre $3.600 \mathrm{~m}$ y $4.200 \mathrm{~m}$.

Los pisos térmicos en el pasado permitieron la delimitación de los páramos de acuerdo con la altitud en las vertientes colombianas. Se denominó páramo bajo a las regiones delimitadas entre las cotas
$3.000 \mathrm{msnm}$ y $3.600 \mathrm{msnm}$, con una temperatura media que variaba entre $8{ }^{\circ} \mathrm{C}$ y $11{ }^{\circ} \mathrm{C}$; este fue denominado también piso térmico muy frío. El páramo alto lo comprenden las tierras localizadas entre las cotas $3.600 \mathrm{msnm}$ y $4.500 \mathrm{msnm}$ (temperatura del aire entre $3{ }^{\circ} \mathrm{C}$ y $8{ }^{\circ} \mathrm{C}$ ) (IGAC, 1992); a su vez, se denomina piso térmico extremadamente frío y precede a los nevados.

Los límites de cota para los páramos en zonas de alta montaña y sus valores de temperatura asignados difieren de un autor a otro; ejemplo de ellos son Cuatrecasas (1958), 
Reyes et al. (1995), Rangel, Lowy \& Cjeef (1995), IDEAM (2010, 2005a) e IGAC (2014d); una muestra de ello es que entre estos dos últimos hay una diferencia de $0,5^{\circ} \mathrm{C}$ por piso térmico.

El enfoque biogeográfico de Zonas de Vida propuesto por Holdridge fue adoptado en Colombia como una clasificación climática en la cual había correspondencia entre estas y el clima (IGAC, 1997); información cartográfica que se aplicó sin inconvenientes a la escala original 1:500.000. Sin embargo, cuando se detalla a escala 1:100.000 surgen incoherencias especialmente cuando se establecen relaciones con el clima de cada cabecera municipal; por tal motivo, a partir del año 2000 comienzan a utilizarse para el análisis climático en los estudios generales de suelos los datos meteorológicos procedentes de las cabeceras municipales. Esto causa cambios en las delineaciones del clima, y pueden observarse en el estudio de suelos del departamento de Cundinamarca (IGAC, 2000).

En los años noventa surgen en Colombia las políticas de planificación del uso del territorio, y se da inicio a las aplicaciones de los le- vantamientos de suelos con nuevos enfoques, tales como el análisis fisiográfico y la ecología del paisaje; la diferencia entre fisiografía y ecología radica en que para la primera el clima es una característica del paisaje, y para la segunda es un producto de la dinámica de la cobertura vegetal (Botero et al., 1997); en las dos aplicaciones se mantiene la semejanza entre pisos térmicos y zonas de vida. Durante el mismo año se incluye el método de Thortwaite, que define el índice hídrico a partir del análisis de humedad y aridez (Villota, 1997).

Con la implementación de los sistemas de información geográfica y el desarrollo tecnológico del software, se incrementa la utilización de datos climáticos georreferenciados y la aplicación de técnicas kriging para definir las unidades climáticas, lo cual representó un avance en la zonificación climática general a escala 1:100.000 (Castro, 2010).

Las técnicas kriging suponen variables climáticas que suceden en un área con poca influencia externa (áreas isotrópicas); la técnica puede utilizarse en espacios pequeños y de relieve plano, alejados de cuerpos de aguas con poca inci- 
dencia de vientos y sin efectos ocasionados por el relieve, tales como el efecto Foehn. Para ampliar su aplicación a escalas intermedias y detalladas es necesario incluir técnicas co-Kriging, en las cuales se adiciona la localización geográfica del dato climático y los modelos digitales de elevación.

El mapa es una representación cartográfica de la realidad, cuya validez se obtiene a través de la cantidad y de los tipos de variables que intervienen en su elaboración. Algunos usuarios de cartografía temática no se convencen de un producto cuando no encuentran en él lo que ellos saben que existe; olvidan que los mapas se construyen de variables representativas, y que su validez y aplicabilidad se relaciona con la escala a la cual fue proyectado; no existe una representación multiescalar, ya que los interrogantes que se esperan resolver con los mapas cambian con la escala (Agualimpia y Castro, 2016).

Existen investigaciones sobre la relación altitud y temperatura del aire; algunos de ellos coinciden en que esta última varía $0,65^{\circ} \mathrm{C} / 100$ m (Binkley y Fisher, 2013, y Flórez, 1986, citando a Hufty, 1984, y a Stanescu y Díaz, 1971). Estudios de caso realizados en Colombia confirman que las vertientes de solana (cara de la vertiente hacia el sur) son más cálidas que las vertientes úmbricas, opuestas a la anterior (Hufty, 1984); por tal razón, el factor presenta diferencias locales, siendo más frías las vertientes exteriores de las cordilleras que las interiores, que conforman el valle del río Magdalena. Flórez (1986) obtuvo variaciones de la temperatura aire de $0,8{ }^{\circ} \mathrm{C} / 100 \mathrm{~m}$ a 0,9 ${ }^{\circ} \mathrm{C} / 100 \mathrm{~m}$ por debajo del nivel de condensación y encontró valores de $0,6{ }^{\circ} \mathrm{C} / 100 \mathrm{~m}$ a $0,7^{\circ} \mathrm{C} / 100 \mathrm{~m}$ por encima del nivel de condensación. El gradiente de temperatura del aire en los análisis de clima ha sido un tema de interés para investigadores como Stanescu y Díaz (1971), Eslava et al. (1986 y 1993) y Cortés (1989). Recientemente, Chaves y Jaramillo (1998), citados en Vélez, Poveda y Mesa (2000), realizaron análisis de regresión lineal para hallar la dependencia de temperatura del aire con la altura, y trabajaron con 1.002 datos procedentes de estaciones meteorológicas.

\section{Materiales y métodos}

Para el desarrollo de esta investigación se realizaron trabajos de campo y generación de cartografía, que 
fue validada a través de visita poscampo. Los elementos utilizados fueron: termómetros LCD digitales con sensor de acero y rango amplio $\left(-50{ }^{\circ} \mathrm{C}\right.$ a $\left.300{ }^{\circ} \mathrm{C}\right)$, GPS, cartografía básica escala 1:100.000, cámara fotográfica digital, palines y palas, mapa digitalizado de zonas de vida (Espinal, 1978), modelo digital del terreno SRTM 30 m (USGS, 2014), computador personal HP $12 \mathrm{MB}$ RAM, software Arc Gis 10.1 y formatos de captura de información en Excel, entre otros elementos.

La zona de estudio se delimitó a partir de la cota $2.800 \mathrm{msnm}$ hasta las cumbres y abarcaba varios complejos de páramos identificados por el Instituto de Investigación en Recursos Biológicos Alexander Von Humboldt (2007). La zona incluye las vertientes oriental y occidental en un sector de la cordillera Oriental, cuyas coordenadas extremas son: norte: $7^{\circ} 53^{\prime} 19,656^{\prime \prime}$, sur: $5^{\circ}$ 6' 28,728", oeste: $73^{\circ} 39^{\prime} 20,884^{\prime \prime}$ y este: $71^{\circ} 59^{\prime} 35,987^{\prime \prime}$. El sector de interés de esta cordillera tiene una longitud norte-sur de $372 \mathrm{~km}$ y abarca desde el Páramo de Santurbán, en Norte de Santander, hasta el complejo de páramos Tota-Bijagual-Mamapacha, en Boyacá, y un ancho máximo de $51,8 \mathrm{~km}$ en el páramo de Cocuy (Instituto de
Investigación en Recursos Biológicos Alexander Von Humboldt, 2007).

El procedimiento que se presenta a continuación es producto del grupo de Investigación PROGASP, de la Universidad Distrital Francisco José de Caldas (Agualimpia y Castro, 2016) y sirvió como base en la delimitación de los suelos de los páramos de la cordillera Oriental durante el estudio semidetallado de suelos realizado por la Subdirección de Agrología del Instituto Geográfico Agustín Codazzi para el Instituto Alexander Von Humboldt.

Durante el reconocimiento de suelos realizado en los meses de septiembre y diciembre de 2013 se tomó la temperatura estabilizada del suelo, definida por la USDA-NRCS (2014), a $50 \mathrm{~cm}$ de profundidad, y la temperatura del aire a $150 \mathrm{~cm}$ de la superficie del suelo debajo de las coberturas vegetales; los valores de temperatura a exposición directa al sol no se consideraron, debido a que representaban diferencias muy altas según Binkley y Fisher (2013). 


\subsection{Submodelo de campo}

Se realizaron 3.040 observaciones distribuidas en el paisaje, a diferentes altitudes y entre las 7 de la mañana y las 2 de la tarde, en las cuales se tomó la temperatura del suelo y la temperatura bajo las coberturas vegetales.

La información de coordenadas, hora de lectura y temperaturas del aire y del suelo se registraron en formatos de papel y luego se digitaron en una base de datos en Excel; se armonizaron los datos y con ellos se realizó un análisis comparativo entre temperatura del suelo y temperatura bajo las coberturas vegetales naturales.

Los datos de temperaturas del aire y del suelo se organizaron según la hora del día para establecer valores promedio y valores ponderados; los primeros fueron calculados para datos tomados a intervalos de 5 minutos, y los ponderados se obtuvieron según la cantidad de datos de temperatura registrados a esa hora del día (Tabla 2).

Tabla 2. Descripción de la diferencia entre temperatura del aire y temperatura del suelo $\left({ }^{\circ} \mathrm{C}\right)$.

\begin{tabular}{|l|c|c|c|c|c|c|c|}
\hline ESTADÍSTICA & $\begin{array}{c}\mathbf{8 : 0 0} \\
\text { a.m. }\end{array}$ & $\begin{array}{c}\mathbf{9 : 0 0} \\
\text { a.m. }\end{array}$ & $\begin{array}{c}\mathbf{1 0 : 0 0} \\
\text { a.m. }\end{array}$ & $\begin{array}{c}\mathbf{1 1 : 0 0} \\
\text { a.m. }\end{array}$ & $\begin{array}{c}\mathbf{1 2 : 0 0} \\
\text { m. }\end{array}$ & $\begin{array}{c}\mathbf{1 : 0 0} \\
\text { p.m. }\end{array}$ & $\begin{array}{c}\mathbf{2 : 0 0} \\
\text { p.m. }\end{array}$ \\
\hline Valor ponderado & 1,25 & 1,69 & 2,14 & 1,89 & 3,81 & 1,56 & 1,30 \\
\hline Valor de la media & 1,23 & 1,93 & 2,25 & 1,89 & 3,87 & 0,99 & 1,30 \\
\hline Desviación estándar & 2,12 & 2,27 & 2,51 & 2,63 & 3,12 & 2,26 & 1,56 \\
\hline $\begin{array}{l}\text { Coeficiente correlación } \\
\text { entre temperatura del } \\
\text { aire y del suelo }\end{array}$ & 0,96 & 0,97 & 0,94 & 0,97 & 0,97 & 0,97 & 0,99 \\
\hline
\end{tabular}

Fuente: Elaboración propia.

Clasificar el régimen de temperatura requiere únicamente valores medios anuales, para definir la clase taxonómica; por ello, el procedimiento estadístico consistió en registrar la desviación de los valores medios y el coeficiente de correla- ción entre la temperatura del suelo y la temperatura del aire registrada bajo las coberturas naturales. Según lo anterior, a través del "submodelo de campo" se encontró que en la zona de la cordillera Oriental 
la temperatura del suelo es $1,9{ }^{\circ} \mathrm{C}$ mayor que la temperatura del aire. El procedimiento para delinear el clima edáfico a partir de isotermas se plantea como solución al problema de representación del régimen de temperatura; para el caso de la cordillera Oriental, los valores promedio de temperatura corresponden a todos los tipos de suelos. La escala a la cual se quiere llegar con esta propuesta es la semidetallada, por tanto, es necesario aplicar otros procedimientos cuando se quiere alcanzar una escala detallada o ultradetallada.

La segunda parte del procedimiento se relaciona con las diferencias en el gradiente de temperatura en las dos vertientes de la cordillera Oriental; para este caso se identificó la línea divisoria de aguas, utilizando el mapa de cuencas hidrográficas suministrado por la oficina de sistemas de información de la Corporación Autónoma Regional de Cundinamarca. La identificación de las cuencas hidrográficas se hizo a partir del modelo digital del terreno SRTM (USGS, 2014), el mismo que se utiliza para generar la información raster de temperatura.
Se obtuvo información de la temperatura del suelo realizada para cada vertiente, se realizó la correlación con la altitud y se hizo el análisis de correlación lineal para datos localizados en vertientes externas e internas, para lo cual se generaron las funciones Solana y Umbría.

Solana se refiere a la vertiente montañosa que recibe mayor cantidad de horas sol y su equivalente en radiación solar; para el caso, son las vertientes occidentales, las que, a su vez, también se componen de abundantes fragmentos de rocas, por localizarse en su mayoría laderas erosionales. La región de Umbría es aquella vertiente montañosa que recibe menor cantidad de horas luz y mayor cantidad de vientos, por presentarse en espacios abiertos (vertiente oriental de la cordillera Oriental). Lo anterior coincide con Sturm y Rangel (1985), quienes encontraron que en la vertiente oriental de la cordillera Oriental (Umbría) cae mayor cantidad de lluvias que en la vertiente occidental (Solana).

Las regresiones para las vertientes quedaron así: 
Solana: $\mathrm{Y}=-0,0073 \mathrm{X}+33,225$

Umbria: $Y=-0,0061 X+26,400$
Ecuación 1

Ecuación 2

Donde: $\mathrm{Y}=$ Temperatura del aire, en ${ }^{\circ} \mathrm{C}$ $\mathrm{X}=$ Altitud, en metros

La Tabla 3 muestra la variación de las temperaturas del aire de acuerdo con la orientación de la vertiente; según el análisis de regresión lineal aplicada a los 3.040 datos tomados en campo, se determinó que en la vertiente de Solana hay un gradiente térmico de $0,73{ }^{\circ} \mathrm{C} / 100 \mathrm{~m}$, y en la vertiente de Umbría el gradiente desciende a $0,61{ }^{\circ} \mathrm{C} / 100 \mathrm{~m}$.
En Solana, al ascender en la montaña desde $3.000 \mathrm{~m}$ hasta $3.700 \mathrm{~m}$ hay un descenso de temperatura de $5,1{ }^{\circ} \mathrm{C}$, mientras que para este mismo ascenso por la vertiente de Umbría la temperatura desciende en menor proporción $\left(4,3{ }^{\circ} \mathrm{C}\right)$; esto demuestra que cuando hay temperaturas bajas la proporción de descenso es menor.

Tabla 3. Coeficientes para región de páramo localizada en la cordillera Oriental.

\begin{tabular}{|l|c|c|c|c|}
\hline \multicolumn{5}{|c|}{ TEMPERATURAS MEDIAS } \\
\hline $\begin{array}{c}\text { Región } \\
\text { paramuna }\end{array}$ & $\begin{array}{c}\text { Temperatura } \\
\text { índice }\end{array}$ & $\begin{array}{c}\text { Coeficiente } \\
\text { temperatura } \\
\text { máxima }\end{array}$ & $\begin{array}{c}\text { Temperatura a } \\
\mathbf{3 . 0 0 0} \text { msnm }\left({ }^{\circ} \mathbf{C}\right)\end{array}$ & $\begin{array}{c}\text { Temperatura a } \\
\mathbf{3 . 7 0 0 ~ m s n m ~}\left({ }^{\circ} \mathbf{C}\right)\end{array}$ \\
\hline Solana & 33,225 & 0,0073 & 11,3 & 6,2 \\
\hline Umbría & 26,400 & 0,0061 & 8,1 & 3,8 \\
\hline
\end{tabular}

Fuente: Elaboración propia.

Las heladas que particularizan las áreas de páramo también se distinguen en las vertientes de Solana y Umbría. En Solana hay mayor probabilidad de que se presenten heladas a causa de la radiación, porque hay mayor oportunidad de alcanzar descensos de temperatura durante la noche en tiempos de cielo despejado; mientras que en las vertientes de Umbría es posible que se presenten heladas por advección, debido a la llegada de masas de aire frío desde regiones nevadas (Seoánez, 2002). 


\subsection{Isotermas del suelo}

Los registros de temperatura se tomaron aplicando el mismo protocolo durante sesenta días y en las dos vertientes de la cordillera. El número de repeticiones registradas a la misma hora del día y los datos promediados dentro de los cinco minutos permitieron obtener un dato promedio aproximado.

El SRTM de 30 m puede contener algunos errores en altitud que son no significativos, pues las coberturas de páramo que podrían aumentar el relieve no exceden los $7 \mathrm{~m}$; además, el gradiente de temperatura encontrado para las dos vertientes es menor a un grado en $100 \mathrm{~m}$ de altitud, y un dato así no altera en gran medida el resultado que se quiere obtener a esta escala. Pruebas realizadas con el modelo digital del terreno de 10 metros no mostraron diferencias significativas al modelo digital de $30 \mathrm{~m}$ utilizado en la presente investigación (Castro y Agualimpia, 2016). El modelo climático se sintetiza a través de los pasos descritos en la Figura 1.

\subsection{Pasos del modelo climático}

- Se escoge el área montañosa que se va a trabajar (sector de la cor- dillera Oriental), se toma la cota de $2700 \mathrm{msnm}$, como límite inferior a trabajar.

- Se divide la zona escogida en vertientes, utilizando la línea divisoria de agua, para observar las diferencias de temperatura del aire entre vertientes.

- A cada una de las vertientes se le toman registros en campo de temperatura del aire.

- Para cada vertiente se obtiene el modelo de elevación.

- Los datos de registro y el modelo se armonizan o preparan para la modelación de variables. Este paso consiste en realizar la función de extracción de dato de la altitud en el modelo digital de elevación para cada punto de temperatura referenciada en campo (3.040 observaciones). Los datos de altitud con el GPS y con el modelo presentan diferencias y deben ajustarse a la precisión que tenga el modelo digital de elevación.

- Cuando se tienen estandarizados los datos de temperatura y altura se realiza un análisis de correlación entre estas variables.

Se obtiene el valor diferencia entre temperaturas del aire y del suelo 
mediante la aplicación del submodelo climático de campo, que tiene tres pasos:

1. En el área de estudio se toman datos de temperatura del aire a $150 \mathrm{~cm}$ debajo de las coberturas vegetales y a $50 \mathrm{~cm}$ de profundidad en el suelo, cara opuesta al sol.

2. Se obtiene registro de las coordenadas y de la hora en que se tomaron los datos de temperatura del aire y del suelo.

3. Mediante análisis estadístico simple se halla el valor de diferencia entre temperaturas del suelo y del aire.

4. Con el submodelo se estableció que en la cordillera Oriental la temperatura del suelo es superior a la del aire en $1,9^{\circ} \mathrm{C}$.

El modelo climático continuó así:

- Los datos de temperatura del aire, armonizados con el modelo digital de elevación, se correlacionan con la altitud y se halla el modelo matemático lineal, que consiste en encontrar la función de la variable independiente temperatura con la dependiente altura.

- La función lineal se ingresa en el sistema con una herramienta
(Raster calculator) para generar el raster de temperatura; la herramienta permite asignar un valor de temperatura del aire a cada altura, según el modelo matemático hallado en el paso anterior.

- Se genera una reclasificación de la temperatura del aire, sumándole a cada celda del raster de temperatura obtenido a través del modelo lineal el valor de $1,9^{\circ} \mathrm{C}$, que es la diferencia encontrada entre las temperaturas del suelo y del aire. El resultado es un raster de temperatura del suelo.

- Se crean clases de temperatura del suelo asociada a rangos $<1,5$ ${ }^{\circ} \mathrm{C}$, de $1,5^{\circ} \mathrm{C}$ a $4{ }^{\circ} \mathrm{C}$, de $4{ }^{\circ} \mathrm{C}$ a 8 ${ }^{\circ} \mathrm{C}$ y de $8{ }^{\circ} \mathrm{C}$ a $12{ }^{\circ} \mathrm{C}$; los rangos proceden de la clave taxonómica de suelos (USDA-NRCS, 2014).

- Cada rango de temperaturas del suelo corresponde a una clase taxonómica del suelo (gélico, cryico, isofrígido); el límite inferior corresponde al régimen de temperatura de suelos isomésico y separa las tierras del páramo bajo de las tierras de piso térmico frío. 


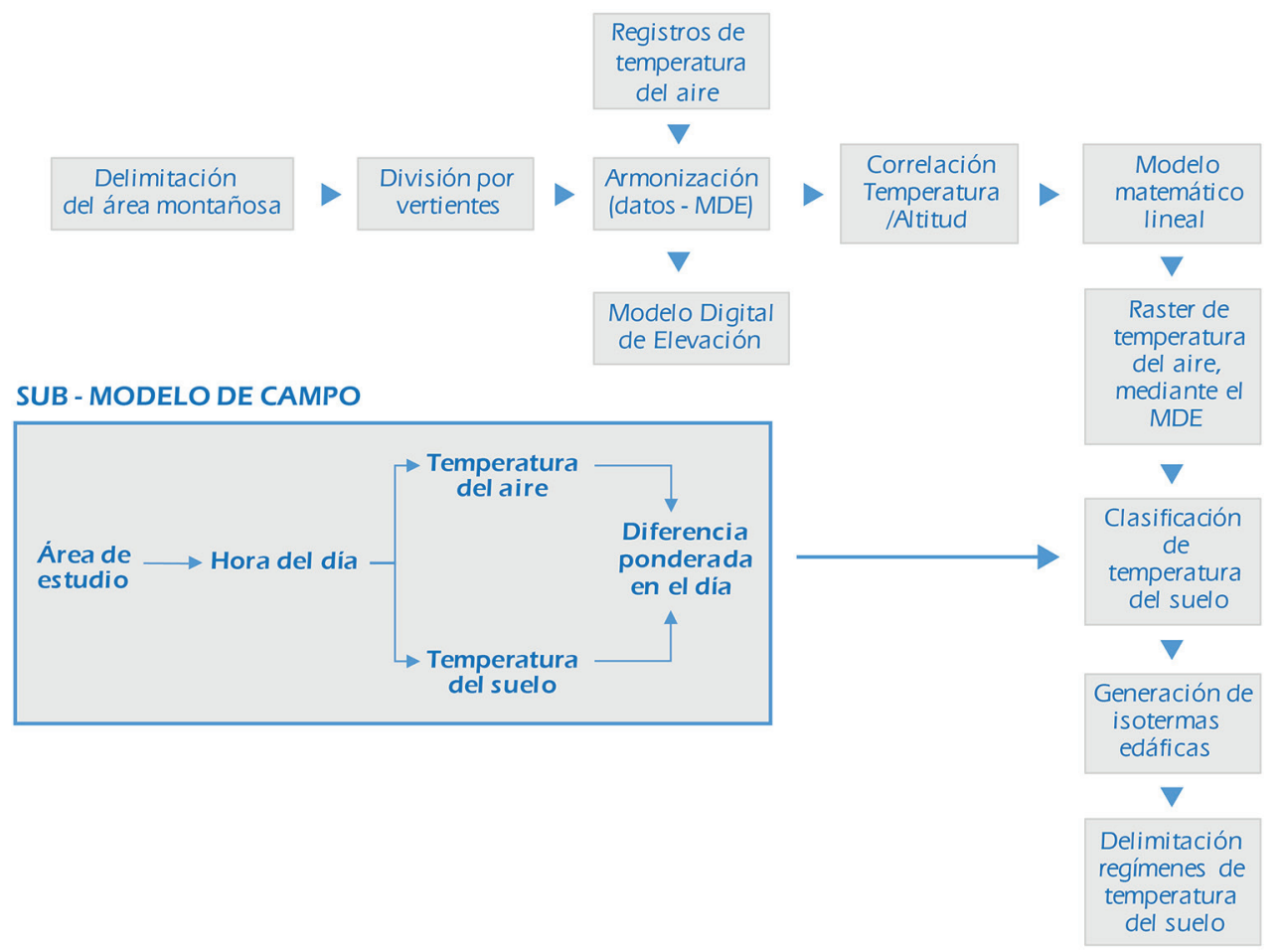

Figura 1. Modelo para generar isotermas del suelo y ajustar la taxonomía Fuente: Elaboración propia.

Las isolíneas de temperatura del la cordillera Oriental, lo cual indisuelo permiten realizar una re- ca que los límites de los regímenes presentación más detallada de los de temperatura no corresponden a regímenes de temperatura corres- los establecidos en los manuales de pondientes mediante un método levantamiento de suelos de Colomque proviene de datos realizados bia. El procedimiento basado en el en campo y no de una simple cota modelo climático permitió sugeque separa los pisos térmicos.

\section{Resultados}

Se encontraron diferencias en el gradiente de temperatura de las vertientes oriental y occidental de rir nuevos límites para el páramo bajo, definiendo la cota inferior a los $3.010 \mathrm{~m}$ en la vertiente oriental y a $3.445 \mathrm{~m}$ en la vertiente occidental; Caldas (1802, citado por IDEAM, 2005a) había establecido que la diferencia en los pisos tér- 
micos podría ser de $200 \mathrm{~m}$ hacia arriba o hacia abajo; con los datos tomados en campo puede darse una aproximación escalar basada en el cambio climático y, específicamente, en un incremento en el rango de incertidumbre con relación a la altitud, ya que al ajustar las cotas establecidas para los regímenes térmicos del suelo se pueden revisar los límites de los páramos colombianos.

La diferencia en el gradiente de temperatura puede representar cambios en el rango de altitud de los pisos térmicos para cada vertiente. Al comparar fuentes antiguas sobre pisos térmicos se observó alguna incertidumbre en la definición de las cotas de altitud; esto afecta directamente la delimitación de los regímenes de temperatura del suelo; Eslava, López y Olaya (1986) reportan que la diferencia positiva o negativa entre pisos térmicos puede ser $400 \mathrm{~m}$ de altitud. Los manuales de suelos desconocen esta variación, y en estudios de suelos se aplicaban sin distinción las mismas altitudes de pisos térmicos y equivalencia en regímenes de temperatura del suelo.

Para explicar por qué el dato de temperatura del aire y del sue- lo tomado en campo muestra una vertiente caliente (Solana) y otra vertiente fría (Umbría), se puede argumentar que la vertiente occidental (interna) está expuesta a mayor radicación solar (IDEAM, 2005b), tiene menor circulación de vientos y se encuentra en la misma dirección del Cañón del Chicamocha, sitio que guarda menos humedad y tiene altos índices de evapotranspiración (Espinal, 1978); esto pudo contribuir al ascenso de calor por la vertiente; mientras que la vertiente de Umbría está expuesta a mayor acción de los vientos y tiene menor tiempo de exposición directa del sol, debido a la constante nubosidad (IDEAM, 2005b).

Para probar la consistencia de los datos tomados en campo se realiza el análisis comparativo de gradientes térmicos con las formaciones vegetales (Espinal, 1978).

En la Figura 2 se observa que la vertiente oriental tiene mayor cantidad de lluvias, lo cual indica alta condensación de aguas, y puede verificarse por la localización en esta vertiente de formaciones vegetales de condición muy húmeda y pluvial (Espinal, 1978); la vertiente oriental es más fría y debe su temperatura del aire y del suelo a la 
presencia de vegetación abundante $\mathrm{y}$, especialmente, a la humedad ambiental. La vertiente occidental de este sector de la cordillera Oriental guarda menos humedad y su vertiente es más corta, e incide en una gradiente de temperatura del aire más alto $\left(0,73{ }^{\circ} \mathrm{C} / 100 \mathrm{~m}\right)$, en comparación con la vertiente oriental, cuyo gradiente es de 0,61 ${ }^{\circ} \mathrm{C} / 100 \mathrm{~m}$.
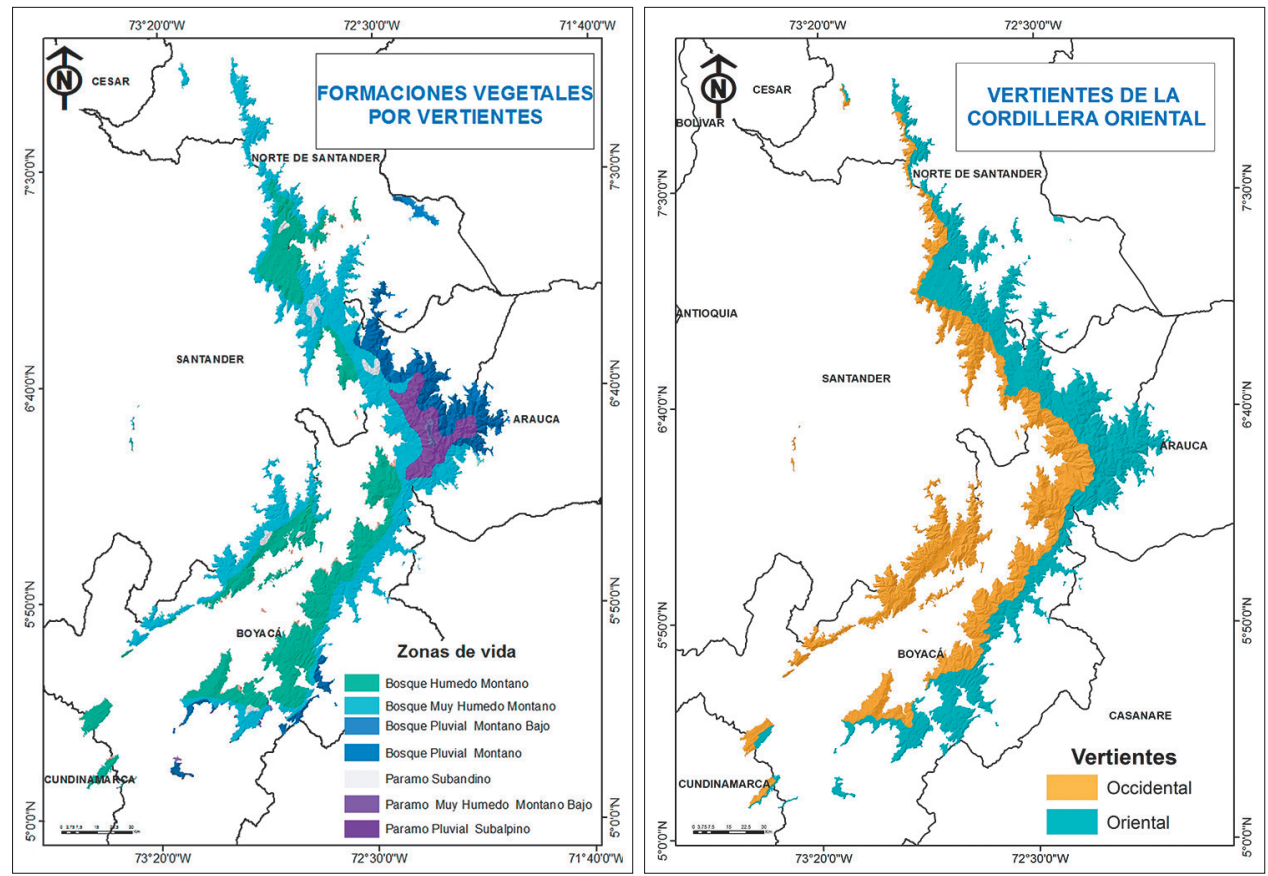

Figura 2. Comparación entre vertientes y zonas de vida

Fuente: Elaboración propia con base en Espinal (1978).

La proximidad de climas secos en las zonas bajas, caso de los enclaves secos, influye en el desplazamiento de las cotas designadas para los pisos térmicos en manuales de levantamiento de suelos, lo mismo que la presencia de climas húmedos en las zonas cálidas causa un descenso brusco de las isotermas edáficas.
Para dar a conocer los avances en la delimitación de regímenes de temperatura del suelo se presenta un análisis comparativo entre el método tradicional con el que se define el régimen térmico del suelo y la propuesta de generar los regímenes de temperatura del suelo a partir de isotermas del suelo. 
El método tradicional consiste en reclasificar cada piso térmico, según una franja altitudinal predeterminada, en un régimen térmico del suelo; en ese sentido, se parte del supuesto de que, en la zona estudiada, el piso frío corresponde a un régimen térmico isomésico del suelo, y el piso extremadamente frío, a un régimen isofrígido (Fi- gura 3). En contraste, se presenta la propuesta de realizar la delimitación del clima mediante isotermas del suelo, en la cual se contempla el gradiente de temperatura por vertiente y una altitud de páramo dependiente de la temperatura del suelo del sistema montañoso de la cordillera Oriental.
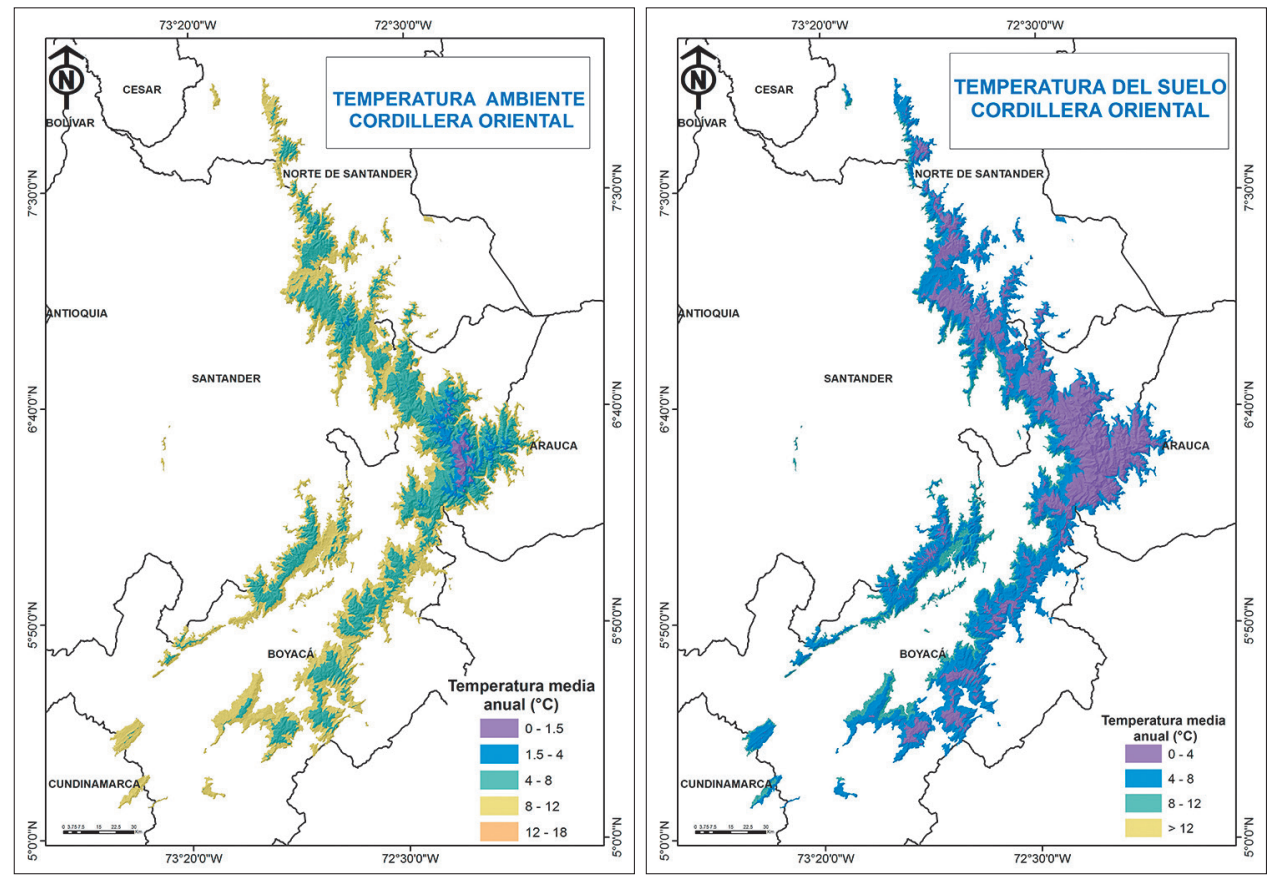

Figura 3. Comparación entre temperatura bajo coberturas vegetales y temperatura del suelo

Fuente: Elaboración propia.

Cuando se aplica el método tradicional de reclasificar el piso térmico según las cotas de altitud establecidas en manuales, y con ese resultado se define el régimen de temperatura del suelo, las isotermas menores a $4{ }^{\circ} \mathrm{C}$, que representan el superpáramo, encierran un área menor que cuando se aplica el método propuesto, basado en 
las isotermas del suelo; en otros términos, con el método basado en isotermas del suelo se identifica mayor extensión en áreas de superpáramo (57.180 ha), que corresponden a régimen térmico cryico. Lo anterior se debe a que la vertiente fría (Umbría) es más larga que la caliente (Solana). El límite bajo obtenido para la vertiente Solana es de $4.000 \mathrm{msnm}$, y para Umbría, de 3,680 msnm.

En manuales de reconocimiento de suelos, del Instituto Geográfico (IGAC, 2014a, b y c), y en la clave taxonómica más reciente del Departamento de Agricultura de los Estados Unidos (USDA-NCRS, 2014), la temperatura del suelo con valores entre $4{ }^{\circ} \mathrm{C}$ y $8{ }^{\circ} \mathrm{C}$ corresponde al régimen de temperatura isofrígido, que representa sectores fríos localizados en la zona intertropical. Aunque la taxonomía del suelo (USDA-NCRS, 2014) define en el mismo régimen de temperatura cryico en Colombia el rango para temperaturas del suelo entre $4{ }^{\circ} \mathrm{C}$ y $8{ }^{\circ} \mathrm{C}$, el clima extremadamente frío tiene alta biodiversidad y corresponde al páramo propiamente dicho (Cuatrecasas, 1958), y los límites serían, en Solana, altitud entre 3.445 y $4.000 \mathrm{msnm}$, y en Umbría, entre 3.010 y 3.680 msnm.

La razón por la cual se propone delimitar el páramo con base en isotermas del suelo $\mathrm{y}$, específicamente, por los regímenes de temperatura, es la falta de consenso entre varios autores.

En la alta montaña de la cordillera Oriental, si no se cuenta con información de la temperatura del suelo, se puede trabajar mediante la correlación de la temperatura del aire y la altitud de estaciones en esta vertiente y la adición del valor $1,9{ }^{\circ} \mathrm{C}$, para generar el dato de la temperatura del suelo, tal como se presentó en el modelo (Figura 1).

En la Tabla 4 se presenta la delimitación de cotas de acuerdo con la temperatura del aire reportada en manuales colombianos (IGAC, 2014d y 2014a); con la clasificación de pisos térmicos según la temperatura del aire se notan variaciones en las cotas; no hay, además, una correspondencia entre pisos térmicos y regímenes térmicos, porque algunos valores de isotermas del suelo están fuera del rango establecido en la clave taxonómica (USDA- NCRS, 2014). 
Tabla 4. Ajuste de la temperatura del suelo por piso térmico de acuerdo con las vertientes de Solana y Umbría

\begin{tabular}{|c|c|c|c|c|c|}
\hline Piso térmico & $\begin{array}{c}\text { Isotermas } \\
\text { del aire } \\
{ }^{\mathbf{}} \mathbf{C}\end{array}$ & $\begin{array}{c}\text { Isotermas } \\
\text { del suelo } \\
{ }^{\mathbf{~}} \mathbf{C}\end{array}$ & $\begin{array}{c}\text { Cotas } \\
\text { ajustadas } \\
\text { (Solana) }\end{array}$ & $\begin{array}{c}\text { Cotas } \\
\text { ajustadas } \\
\text { (Umbría) }\end{array}$ & $\begin{array}{c}\text { Régimen } \\
\text { térmico }\end{array}$ \\
\hline Subnival y nival & Menor a 4 & Menor a 5,9 & $>4260$ & $>3999$ & Cryico \\
\hline Subnival y nival & 4 a 8 & 5.9 a 9,9 & $3710-4260$ & $3320-3999$ & Cryico \\
\hline $\begin{array}{c}\text { Extremadamente } \\
\text { frío }\end{array}$ & 8 a 12 & 9.9 a 13.9 & $3170-3710$ & $2680-3320$ & Isofrígido \\
\hline Muy frío & 12 a 18 & 13,9 a 19,9 & $2345-3170$ & $1690-2680$ & Isomésico \\
\hline
\end{tabular}

Fuente: Elaboración propia.

En la Tabla 4 se muestra cómo en la vertiente de Solana la temperatura del aire decae rápidamente, en comparación con la vertiente de Umbría; es decir, en un ascenso de $638 \mathrm{~m}$ promedio por la vertiente Solana, la temperatura del aire baja $4{ }^{\circ} \mathrm{C}$, y la vertiente Umbría, para descender los mismos $4{ }^{\circ} \mathrm{C}$ de temperatura del aire, requiere un ascenso más largo (767 m). En la vertiente occidental (Solana) la cota del régimen de temperatura del suelo es $390 \mathrm{~m}$ más alta que en la vertiente oriental (Umbría). La aplicación de gradientes de temperatura a cada vertiente de la cordillera permite definir las cotas límite de regímenes de temperatura del suelo; los registros de temperatura se obtuvieron durante el inicio de la temporada de lluvias en esta región de la cordillera, y es posible que esas cotas desciendan en las dos vertientes en época seca; sin embargo, en consulta con el autor del Atlas de Radiación Solar (IDEAM, 2005) se admite que la nubosidad es un factor importante en el análisis específico de la temperatura del aire en estas vertientes, ya que la vertiente oriental permanece con nubes casi todo el año, y esto permite entender por qué es más fría; posiblemente, esa condición no permita variaciones significativas en la temperatura promedio anual del aire y del suelo y no sea influyente en los regímenes de temperatura del suelo.

\section{Conclusiones}

No existe en Colombia el procedimiento documentado para determinar los regímenes de temperatura del suelo; los manuales de otros países son poco explícitos en 
la manera de definir isotermas. La ausencia de normas que orienten los procedimientos lleva a la aplicación de criterios variados $\mathrm{y}$, algunas veces, sesgados hacia otros propósitos; es necesario proponer ajustes a las unidades climáticas y evaluar su aplicación a los regímenes de temperatura del suelo.

El procedimiento aplicado a Colombia consiste en dar una valoración de régimen de temperatura del suelo acorde con el piso térmico, sin utilizar geotermómetros y sin ajuste de la distribución de tempe- raturas en el suelo; los regímenes térmicos del suelo se definen a partir de cotas altitudinales preestablecidas.

Se diseñó y probó un modelo climático en un sector de la cordillera Oriental, el cual permitió aportar elementos para mejorar la resolución escalar con la que se delimitan los pisos térmicos, basándose en datos tomados en campo; esto contribuyó a la generación directa de regímenes térmicos a partir de isotermas del suelo.

\section{Referencias}

Agualimpia, Y. y Castro, C. (2016). Propuesta metodológica para la zonificación climática a diferentes escalas en Colombia, con fines de manejo sostenible del territorio. Fase I. Bogotá, D. C.: Centro de Investigaciones de la Universidad Distrital (CIUD). Grupo de Investigación Programa de Gestión Ambiental en Servicios Públicos (PROGASP).

Bartels, S. G. (1984). Los pisos morfoclimáticos de la Sierra Nevada de Santa Marta. En T. Van Der Hammen y P. M. Ruíz (Eds.), La Sierra Nevada de Santa Marta (Colombia). Transepto Buritica - La Cumbre (pp. 99-129). Berlín: Cramer.

Binkley, D. y Fisher, R. (2013). Ecology and Management of Forest Soils. 4a ed.: Colorado State University.

Botero, P., Jiménez, B., Rodríguez, N., Herrera, J., Rodríguez, J., Mendoza, Y. y Pinilla, A. (1997). Estudio fisiográfico de la orinoquía-amazonía colombiana. Revista CIAF, 15(1), $7-62$.

Castro, C. (2010). Instructivo para aplicar procedimientos digitales en la delimitación del clima en áreas homogéneas de tierras para catastro. Uso interno. Instituto Geográfico Agustín Codazzi. 
Cuatrecasas, J. (1958). Aspecto de la vegetación natural de Colombia. Revista Academia Colombiana de Ciencias Exactas, Físicas y Naturales, 10(40), 221-264.

Eslava, J., López, V. y Olaya, G. (1986). Los climas de Colombia (sistema de clasificación Caldas-Lang), Revista Atmósfera, 7, 41-47.

Espinal, S. (1978). Zonas de vida o formaciones vegetales de Colombia. Memoria explicativa sobre el mapa ecológico escala 1:500.000; realizado en el Instituto Geográfico Agustín Codazzi. Subdirección de Agrología. Bogotá, Colombia.

FAO (2012). Food and Agriculture Organization. Guía para la descripción de suelos. Recuperado de http://www.fao.org/3/a-a0541s.pdf

Flórez, A. (1986). Relación altitudinal de la temperatura del suelo y del aire en los Andes centrales colombianos. Instituto Geográfico Agustín Codazzi. Revista Colombia Geográfica, XII(2), 5-39.

Flórez, A. (2003). Colombia: Evolución de sus relieves y modelados. Bogotá: Universidad Nacional de Colombia, UNIBIBLOS.

Hufty, A. (1984). Introducción a la climatología. Barcelona: Ariel Geografía.

IDEAM-Instituto de Hidrología, Meteorología y Estudios Ambientales. (2005a). Atlas Climatológico de Colombia. Bogotá, D.C.

IDEAM. (2005b). Atlas de radiación solar de Colombia. Bogotá, D. C.: Ministerio de Minas y Energía, Ministerio de Ambiente Vivienda y Desarrollo Territorial.

IDEAM. (2010). Sistemas morfogenéticos del territorio colombiano. Bogotá.

IDEAM, IGAC, IAVH, INVEMAR, SINCHI e IIAP. (2007). Ecosistemas continentales, costeros y marinos, Bogotá, D.C.: Imprenta Nacional de Colombia. + 1 CD-ROOM, 37 planchas.

IGAC -Instituto Geográfico Agustín Codazzi. (1981). La relación clima-suelo: Influencia del clima en la formación y características de los suelos. Centro Interamericano de Fotointerpretación. Unidad de Suelos y Agricultura. Bogotá; mimeógrafo.

IGAC (1992). Manual codificado para la descripción de perfiles. Subdirección de Agrología. Bogotá. Uso interno.

IGAC (1997). Metodología para la elaboración de áreas homogéneas con fines catastrales. Bogotá, D. C.: Subdirección de Agrología. Ministerio de Hacienda y Crédito Público.

IGAC (2000). Estudio general de suelos y zonificación de tierras del departamento de Cundinamarca (tres tomos). Bogotá, D.C.: Subdirección de Agrología. 
IGAC (2014a). Metodología de levantamiento de suelos. Bogotá, D.C.: Grupo interno de trabajo Levantamiento de Suelos.

IGAC (2014b). Instructivo códigos para los levantamientos de suelos. Bogotá, D.C.: Grupo interno de trabajo Levantamiento de Suelos.

IGAC (2014c). Guía para elaborar la memoria técnica de un estudio de suelos. Bogotá, D.C.: Grupo interno de trabajo Levantamiento de Suelos.

IGAC (2014d). Zonificación climática. Bogotá, D.C.: Grupo interno de trabajo Levantamiento de Suelos.

IAVH -Instituto de Investigación en Recursos Biológicos Alexander Von Humboldt. (2007). Atlas de páramos de Colombia. Bogotá, D.C.

McKenzie, N., Grundy, M., Webster, R. y Ringrose-Voase, A. (2008). Guidelines for Surveying Soil and Land Resources. 2da. ed. Australia: CSIRO Publishing,

Milo, I., Save, T. y Bennett, W. (1997). Soil Science Simplified. 30va. ed. Estados Unidos: The Iowa State University Press.

Narváez, G. y León, G. (2001). Caracterización y zonificación climática de la región andina. Revista Meteorología Colombiana, 4, 121-126.

Pabón, J., Eslava J. y Gómez, R. (2001). Generalidades de la distribución espacial y temporal de la temperatura del aire y la precipitación en Colombia. Meteorología Colombiana, 4, 47-59.

Reyes, O., Molano, J., González, F., Cortez, A., Rangel, O., Flórez, A., Iriarte, P. y Kraus, E. (1995). El páramo: un ecosistema de alta montaña. Bogotá D.C.: Fundación Ecosistemas Andino y del Medio Ambiente.

Seoánez, M. (2002). Tratado de climatología aplicada a la ingeniería medioambiental. Análisis climático. Uso del análisis climático en los estudios medioambientales. Barcelona, España: Mundi-prensa.

Stanescu, S. y Díaz, J. R. (1971). Estudio preliminar de la temperatura del aire en Colombia. Bogotá D. C.: Servicio Colombiano de Meteorología e Hidrología.

Sturm, H. y Rangel, O. (1985). Ecología de los páramos andinos: una visión preliminar integrada. Bogotá, D. C.: Universidad Nacional de Colombia.

The National Committee on Soil and Terrain (2009). Australian Soil and Land Survey Field Handbook. 3ra ed. Australia: CSIRO Publishing.

\begin{tabular}{l|l}
\hline 60 & Carlos Enrique Castro Méndez, Yolima del Carmen Agualimpia Dualiby, Fernando Sánchez Sánchez
\end{tabular} 
USDA - United States Department of Agriculture- (1993). Soil Survey Manual Agricultural Handbook, No. 18. Recuperado de http://www.nrcs.usda.gov/wps/portal/nrcs/ detail//?cid=nrcs142p2_054262

USDA-NRCS -United States Department of Agriculture-Natural Resources Conservation Service- (2012). Field Book for Describing and Sampling Soils, ver. 3. National Soil Survey Center Natural Resources Conservation Service. U.S. Department of Agriculture (2012).

USDA-NRCS (2014). Keys to Soil Taxonomy. 12va. ed. United States Departament of Agriculture.

USGS (2014). U.S. Department of the Interior | U.S. Geological Survey. Recuperado de http:// srtm.usgs.gov/data/obtainingdata.php.

Vélez, J.; Poveda, G. y Mesa, O. (2000). Balances Hidrológicos de Colombia. Universidad Nacional de Colombia. Facultad de Minas. Posgrado de Aprovechamiento de Recursos Hidráulicos, Medellín, Colombia.

Villota, H. (1997). Una nueva aproximación a la clasificación fisiográfica del terreno. Revista CIAF, 15(1), 83-115. 
Recepción: 18 de junio de 2015

Evaluación: 29 de enero de 2016

Aprobación: 11 de abril de 2016

\begin{tabular}{l|l}
\hline 62 & Carlos Enrique Castro Méndez, Yolima del Carmen Agualimpia Dualiby, Fernando Sánchez Sánchez
\end{tabular} 